\title{
Studies on Synthesis of Some New Sydnone Containing Compounds and their Biological Activities
}

\author{
PIYUSH P. SAVALIYA, VIKUNJANA K. AKBARI and KESHAV C. PATEL ${ }^{*}$ \\ Department of Chemistry, Veer Narmad South Gujarat University, Surat-395007, \\ Gujarat, India \\ akbarivk@yahoo.co.in
}

Received 23 October 2012 / Accepted 12 November 2012

\begin{abstract}
Some new sydnone derivatives 3-[4-(morpholin-4-yl)phenyl]-4-(secondary substituted amino-4-yl methyl)-sydnone 6(a-j), 3-[4-(morpholin-4-yl) phenyl]-4-(secondary substituted amino-4yl sulfonyl) sydnone 8(a-j) were synthesized starting from 4-chloroaniline (1). The characterization of the newly synthesized compounds was established by IR, ${ }^{1} \mathrm{H}$ NMR, ${ }^{13} \mathrm{C}$ NMR and Elemental analysis. The final compounds were tested for their antimicrobial activity against several microbes.
\end{abstract}

Keywords: Sydnone, Mesoionic, Antimicrobial activity

\section{Introduction}

Mesoionic compounds are of special interest among heterocycles due to their unusual structure, chemical properties and synthetic utility ${ }^{1}$. Sydnones are the most important representatives of mesoionic compounds having the 1, 2, 3-oxadiazole skeleton bearing an oxygen atom attached to the 5 position $^{2,3}$. Various elecrophilic substitution reactions such as halogenation ${ }^{4-7}$, nitration $^{8}$, acylation ${ }^{9,10}$, sulphonation ${ }^{11}$ occur at the fourth position of the sydnone ring containing a hydrogen atom ${ }^{12}$. Sydnone derivatives have been the focus of great interest because of their remarkable biological properties ${ }^{13-15}$, such as antibacterial ${ }^{16,17}$, antitumor ${ }^{18,19}$, antifungal $^{20,21}$, antimalarial ${ }^{22-24}$, antiparasitic ${ }^{25}$, analgesic ${ }^{26}$, antioxidant ${ }^{27}$, anti inflammatory ${ }^{28}$. Sydnone derivatives also show significant response of coronary dilation test, collagen induced platelet aggregation inhibition, local anaesthetic, antiwrithing, anticonvulsant, muscle relaxation and moderate cardio tropic activity ${ }^{29}$. Sydnones show liquid crystalline properties ${ }^{30}$. The $N$-methyl sydnones having a high dielectric constant was used as a solvent for lithium battery electrolyte ${ }^{31}$. We planned to undertake the synthesis and characterization of some new sydnone derivatives containing some heterocyclic system at position-4 linked through methylene and sulphonyl bridge with the hope to achieve enhanced biological activity.

\section{Experimental}

Melting points were determined by open capillary method and are uncorrected. The structures of the compounds were confirmed by ${ }^{1} \mathrm{H}$ and ${ }^{13} \mathrm{C}$ nuclear magnetic resonance and 
Fourier transform infrared. ${ }^{1} \mathrm{H}$ NMR spectra were recorded with Bruker Avance II $400 \mathrm{MHz}$ NMR spectrometer at SAIF, Chandigarh, in $\mathrm{CDCl}_{3}$ or DMSO- $d_{6}$ using TMS as internal standard and chemical shifts are expressed in $\delta$ ppm. ${ }^{13} \mathrm{C}$ NMR spectra of the compounds were recorded with a Bruker Avance II $400 \mathrm{MHz}$ NMR spectrometer at SAIF (Sophisticated Analytical Instrument Facilities), Chandigarh. The IR spectra were recorded with a Thermo Scientific Nicolet iS10 FTIR specrophotometer at the Deparetment of Chemistry, Veer Narmad South Gujarat University. Elemental analysis (C, H, N) were performed on Thermo Finnigan EA 1112 Flash Elemental Analyzer at G.N.F.C. (Gujarat Narmada Valley Fertilizer Company Ltd., Bharuch). The progress of reactions and the purity of synthesized compounds were checked by TLC on E-Merck precoated $60 \mathrm{~F}_{254}$ plates and the spotes were examined under short-wave UV light. The synthesis of 3-[4-(morpholin-4-yl) phenyl]-4(secondary substituted amino-4-yl methyl)-sydnone 6(a-j) and 3-[4-(morpholin-4-yl) phenyl]-4-(secondary substituted amino-4-yl sulfonyl) sydnone 8(a-j) are showm in Scheme 1.

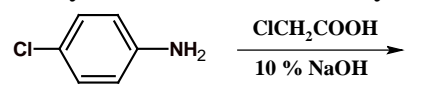

1

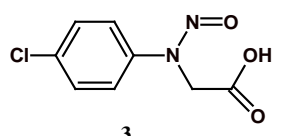

3

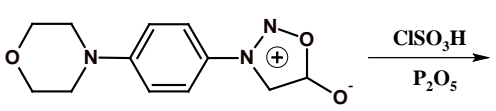

5

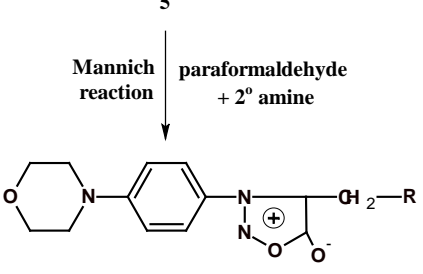

6(a-j)
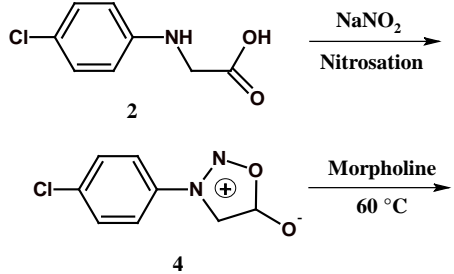

4

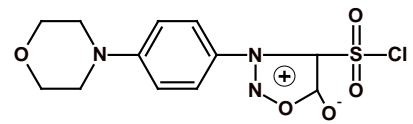

7

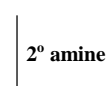

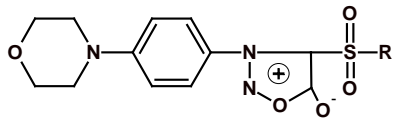

8(a-j)

Scheme 1

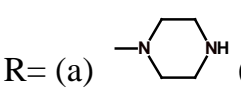

(b)<smiles>CN1CCOCC1</smiles>

(c)

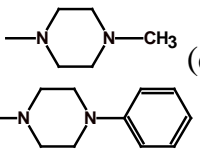

(d)<smiles>CN1CCCCC1</smiles>

(e)<smiles>CN1CCN(C(=O)C[Hg]C=[Ni])CC1</smiles>

(i)<smiles>CN1CCN(C)C(P)C1</smiles>

(j)

\section{General synthesis of intermediates and product}

\section{$\mathrm{N}$-(4-Chlorophenyl) glycine (2)}

4-Chloroaniline ( $0.011 \mathrm{~mol})$ was added to an ice-cooled solution of chloroacetic acid (0.01 mol) and $2 \mathrm{~mL}$ of water which was neutralized by $10 \%$ sodium hydroxide solution. The reaction mixture was refluxed for 20 minutes after cooling to room temperature in an ice bath, $0.5 \mathrm{~g}$ of sodium hydroxide pellet was added and the mixture was extracted with methylene dichloride 
to remove the unreacted aniline. Acidifying the aqueous solution with concentrated $\mathrm{HCl}$ till complete precipitation and recrystallization with ethanol to give compound 2. Yield 80.00\%; mp 145-147 ${ }^{\circ} \mathrm{C}$; IR spectrum (KBr, v, $\left.\mathrm{cm}^{-1}\right)$ : 3323-3278 (OH, NH), 2937, 2937 $\left(\mathrm{CH}_{2}\right), 1705$ (CO); ${ }^{1} \mathrm{H}$ NMR spectrum (DMSO-d $\left.d_{6} \delta \mathrm{ppm}\right): 4.31\left(\mathrm{~s}, 2 \mathrm{H}, \mathrm{CH}_{2}\right), 6.45$ (s, 1H, COOH), 6.56 (s, 1H, NH), 6.90-7.23 (m, 4H, Ar-H); ${ }^{13} \mathrm{C}$ NMR spectrum (DMSO$\left.d_{6}, \delta \mathrm{ppm}\right): 44.98,114.32,123.26,129.10,146.07,172.18$. Anal. calcd. for $\mathrm{C}_{6} \mathrm{H}_{5} \mathrm{~N}_{3} \mathrm{~S}: \mathrm{C}$, 47.66; H, 3.33; N, 27.79; S, 21.21; found: C, 47.55; H, 3.42; N, 27.70; S, 21.32.

\section{$\mathrm{N}$ - Nitroso $\mathrm{N}$-(4-chlorophenyl) glycine (3)}

A cold solution of sodium nitrite $(0.01 \mathrm{~mol})$ in $5 \mathrm{~mL}$ of water was added drop wise to the suspension of $\mathrm{N}$-(4-chlorophenyl) glycine $(0.01 \mathrm{~mole})$ in $40 \mathrm{~mL}$ of water at $0-5{ }^{\circ} \mathrm{C}$ with stirring. After complete addition stirring was continued for $2 \mathrm{~h}$ and keeps it overnight, the reaction mixture was filtered off and the nitroso compound was precipitated by adding concentrated $\mathrm{HCl}$. The product was collected, dried and recrystallised from methanol to furnish compound 3. Yield 78.00\%; mp 104-106 ${ }^{\circ} \mathrm{C}$; IR spectrum $\left(\mathrm{KBr}, v, \mathrm{~cm}^{-1}\right)$ : 3258$2530(\mathrm{OH}), 2925,2857\left(\mathrm{CH}_{2}\right), 1715$ (CO), 1571 (NO); ${ }^{1} \mathrm{H}$ NMR spectrum (DMSO-d ${ }_{6}$, $\delta$ ppm): 5.02 (s, $\left.2 \mathrm{H}, \mathrm{CH}_{2}\right), 6.93-7.48(\mathrm{~m}, 4 \mathrm{H}, \mathrm{Ar}-\mathrm{H}), 11.56(\mathrm{~s}, 1 \mathrm{H}, \mathrm{COOH}) ;{ }^{13} \mathrm{C} \mathrm{NMR}$ spectrum (DMSO-d ${ }_{6}, \delta$ ppm): 49.43, 120.78, 128.31, 130.46, 138.89, 168.24. Anal. calcd. for $\mathrm{C}_{8} \mathrm{H}_{6} \mathrm{ClN}_{3} \mathrm{OS}$ : C, 42.20; H, 2.66; N, 18.46; S, 14.08; found: C, 42.11; H, 2.75; N, 18.34; S, 14.19 .

\section{3-(4-Chlorophenyl) sydnone (4)}

The dried $\mathrm{N}$-nitroso- $\mathrm{N}$-(4-chlorophenyl) glycine and acetic anhydride were taken in ratio of 1: 5 by weight and stirred for $10 \mathrm{~h}$. The solution was poured slowly in to cold water which was very well stirred. The $\mathrm{pH}$ of the content was neutralized with $10 \%$ sodium bicarbonate solution and washed with water and dried. The crude sydnones was recrystallized from benzene-petroleum ether to give compound 4. Yield 98.00\%; mp 140-145 ${ }^{\circ} \mathrm{C}$; IR spectrum $\left(\mathrm{KBr}, v, \mathrm{~cm}^{-1}\right): 3178(\mathrm{CH}), 1750$ (CO); ${ }^{1} \mathrm{H}$ NMR (DMSO-d 6 , $\delta$ ppm): 7.22 (s, $1 \mathrm{H}$, sydnone), 7.55-8.18 (m, 4H, Ar-H); ${ }^{13} \mathrm{C}$ NMR (DMSO-d $d_{6}, \delta$ ppm): 124.12, 128.55, 130.63, 135.22, 140.92, 169.19. Anal. calcd. For $\mathrm{C}_{8} \mathrm{H}_{9} \mathrm{~N}_{5} \mathrm{OS}$ : C, 43.04; H, 4.06; N, 31.37; S, 14.36; found: C, 43.16; H, 4.18; N, 31.25; S, 14.24.

\section{3-[4-(Morpholin-4-yl) phenyl] sydnone (5)}

3-(4-Chlorophenyl) Sydnone $(0.01 \mathrm{~mol})$ and morpholine $(0.01 \mathrm{~mol})$ were refluxed for $24 \mathrm{~h}$. The solution was poured over the crushed ice. The resulting crystals were filtered, rinsed with cold hexane and recrystallized from ethanol to give compound 5. Yield 78.00\%; mp 177-179 ${ }^{\circ} \mathrm{C}$. IR spectrum (KBr, $\left.v, \mathrm{~cm}^{-1}\right)$ : 1754 (CO), $3150(\mathrm{CH}) ;{ }^{1} \mathrm{H}$ NMR $\left(\mathrm{CDCl}_{3}, \delta \mathrm{ppm}\right)$ : 3.17 (t, 4H, -N-CH $)$, 3.64 (t, 4H, -O-CH ${ }_{2}$ ), 6.76-7.73 (m, 5H, Ar-H); ${ }^{13} \mathrm{C}$ NMR (DMSO-d $d_{6}$, $\delta \mathrm{ppm}): 53.51,66.53,114.12,123.19,123.42,126.35,149.58,169.19$. Anal. calcd. for $\mathrm{C}_{12} \mathrm{H}_{15} \mathrm{~N}_{3} \mathrm{O}_{3}$ : C, 57.84; H, 6.06; N, 16.87; found: C, 57.73; H, 6.12; N, 16.82.

\section{Synthesis of compounds $(\mathbf{6} \boldsymbol{a}-\mathbf{j})$}

The mixture of various secondary amine $(0.009 \mathrm{~mol}), 0.25 \mathrm{~g}$ paraformaldehyde and 3-[4(morpholin-4-yl) phenyl] sydnone $(0.003 \mathrm{~mol})$ were added to $10 \mathrm{~mL}$ of acetic acid and $10 \mathrm{~mL}$ ethanol and whole the mixture was heated at $70{ }^{\circ} \mathrm{C}$ for $3 \mathrm{~h}$. After complete the reaction cool it and ethanol was distilled off, $20 \mathrm{~mL}$ of water was added and neutralized with aqeous sodium bicarbonate to afford the crude product. Recrystallization from $95 \%$ ethanol to give compounds 6(a-j). 
3-[4-(Morpholin-4-yl) phenyl]- 4-(piperazin-4-yl methyl)sydnone (6a)

Yield: 65\%; mp 149-151 ${ }^{\circ} \mathrm{C}$; IR spectrum $\left(\mathrm{KBr}, v, \mathrm{~cm}^{-1}\right)$ : $3233(\mathrm{NH}), 2933,2866\left(\mathrm{CH}_{2}\right)$, 1740 (CO); ${ }^{1} \mathrm{H}$ NMR (DMSO- $d_{6}, \delta$ ppm): 1.93 (s,1H, NH), 2.42 (t, 4H, -N-CH $), 2.63$ (t, 4H, $\left.-\mathrm{N}-\mathrm{CH}_{2}\right), 3.23$ (t, $\left.4 \mathrm{H},-\mathrm{N}-\mathrm{CH}_{2}\right), 3.54\left(\mathrm{~s}, 2 \mathrm{H},-\mathrm{CH}_{2}\right), 3.65\left(\mathrm{t}, 4 \mathrm{H},-\mathrm{O}-\mathrm{CH}_{2}\right), 6.89-7.78(\mathrm{~m}, 4 \mathrm{H}$, Ar-H); ${ }^{13} \mathrm{C}$ NMR (DMSO- $d_{6}, \delta$ ppm): 44.56, 51.07, 53.01, 54.46, 66.34, 114.33, 126.35, 128.06, 142.48, 149.67, 168.25. Anal. calcd. for $\mathrm{C}_{17} \mathrm{H}_{23} \mathrm{~N}_{5} \mathrm{O}_{3}$ : C, 59.12; H, 6.71; N, 20.28; found: C, 59.07; H, 6.76; N, 20.21.

\section{3-[4-(Morpholin-4-yl) phenyl]-4-(morpholin-4-yl methyl)sydnone (6b)}

Yield: 69\%; mp 110-112 ${ }^{\circ} \mathrm{C}$; IR spectrum $\left(\mathrm{KBr}, v, \mathrm{~cm}^{-1}\right)$ : 2918, $2840\left(\mathrm{CH}_{2}\right), 1747$ (CO); ${ }^{1} \mathrm{H}$ NMR (DMSO-d $\left.d_{6}, \delta \mathrm{ppm}\right): 2.55$ (t, 4H, $\left.-\mathrm{N}-\mathrm{CH}_{2}\right), 3.25$ (t, 4H, $\left.-\mathrm{N}-\mathrm{CH}_{2}\right), 3.56\left(\mathrm{~s}, 2 \mathrm{H},-\mathrm{CH}_{2}\right)$, 3.65 (t, 8H, -O-CH $)$, 6.90-7.79 (m, 4H, Ar-H); ${ }^{13} \mathrm{C}$ NMR (DMSO- $d_{6}, \delta$ ppm): 47.58, 53.02, 54.49, 66.32, 114.38, 126.42, 128.09, 142.34, 149.63, 168.15. Anal. calcd. for $\mathrm{C}_{17} \mathrm{H}_{22} \mathrm{~N}_{4} \mathrm{O}_{4}$ : C, 58.95; H, 6.40; N, 16.17; found: C, 58.87; H, 6.45; N, 16.23 .

\section{3-[4-(Morpholin-4-yl) phenyl]-4-(1-methylpiperazin-4-yl methyl)sydnone (6c)}

Yield: 55\%; mp 113- $115{ }^{\circ} \mathrm{C}$; IR spectrum (KBr, v, cm $\left.{ }^{-1}\right)$ : 2986-2853 $\left(\mathrm{CH}_{2}, \mathrm{CH}_{3}\right), 1760$ (CO); ${ }^{1} \mathrm{H}$ NMR (DMSO-d $\left.d_{6}, \delta \mathrm{ppm}\right): 2.23\left(\mathrm{~s}, 3 \mathrm{H}, \mathrm{CH}_{3}\right), 2.34\left(\mathrm{t}, 4 \mathrm{H},-\mathrm{N}-\mathrm{CH}_{2}\right), 2.46\left(\mathrm{t}, 4 \mathrm{H},-\mathrm{N}-\mathrm{CH}_{2}\right.$ ), $3.22\left(\mathrm{t}, 4 \mathrm{H},-\mathrm{N}-\mathrm{CH}_{2}\right), 3.54\left(\mathrm{~s}, 2 \mathrm{H},-\mathrm{CH}_{2}\right), 3.63\left(\mathrm{t}, 4 \mathrm{H},-\mathrm{O}-\mathrm{CH}_{2}\right), 6.92-7.84(\mathrm{~m}, 4 \mathrm{H}, \mathrm{Ar}-\mathrm{H}) ;{ }^{13} \mathrm{C}$ NMR (DMSO- $d_{6}, \delta$ ppm): 46.91, 48.42, 53.01, 54.39, 56.28, 66.32, 114.12, 126.45, 128.14, 142.39, 149.73, 168.18. Anal. calcd. for $\mathrm{C}_{18} \mathrm{H}_{25} \mathrm{~N}_{5} \mathrm{O}_{3}$ : C, 60.15; H, 7.01; N, 19.48; found: C, 60.07; H, 6.93; N, 19.43.

\section{3-[4-(Morpholin-4-yl) phenyl]-4-(piperidin-4-yl methyl)sydnone (6d)}

Yield: 59\%; mp 123-125 ${ }^{\circ} \mathrm{C}$; IR spectrum $\left(\mathrm{KBr}, v, \mathrm{~cm}^{-1}\right)$ : 2921, $2862\left(\mathrm{CH}_{2}\right), 1748(\mathrm{CO}) ;{ }^{1} \mathrm{H}$ NMR (DMSO- $d_{6}, \delta$ ppm): 1.51-1.59 (m, 6H, 3CH $), 2.43\left(\mathrm{t}, 4 \mathrm{H},-\mathrm{N}-\mathrm{CH}_{2}\right), 3.21(\mathrm{t}, 4 \mathrm{H},-\mathrm{N}-$ $\mathrm{CH}_{2}$ ), 3.54 (s, $\left.2 \mathrm{H},-\mathrm{CH}_{2}\right), 3.65$ (t, 4H, - $\left.\mathrm{O}-\mathrm{CH}_{2}\right), 6.93-7.87$ (m, 4H, Ar-H); ${ }^{13} \mathrm{C}$ NMR (DMSO$d_{6}, \delta$ ppm): 24.29, 24.89, 49.33, 53.00, 54.35, 66.30, 114.27, 126.35, 128.33, 142.32, 149.88, 168.23. Anal. calcd. for $\mathrm{C}_{18} \mathrm{H}_{24} \mathrm{~N}_{4} \mathrm{O}_{3}$ : C, 62.77; H, 7.02; N, 16.27; found: C, 62.69; H, 6.96; N, 16.33 .

\section{3-[4-(Morpholin-4-yl) phenyl]-4-(1-acetylpiperazin-4-yl methyl)sydnone (6e)}

Yield: 61\%; mp 151-154 ${ }^{\circ} \mathrm{C}$; IR spectrum $\left(\mathrm{KBr}, v, \mathrm{~cm}^{-1}\right)$ : 2936, $2854\left(\mathrm{CH}_{2}\right), 1745$ (CO), 1684 (CO); ${ }^{1} \mathrm{H}$ NMR (DMSO-d $d_{6}, \delta$ ppm): 2.23 (s, 3H, $\mathrm{CH}_{3}$ ), 2.49 (t, $4 \mathrm{H},-\mathrm{N}_{-} \mathrm{CH}_{2}$ ), 3.19 (t, $\left.4 \mathrm{H},-\mathrm{N}-\mathrm{CH}_{2}\right), 3.42$ (t, 4H, $-\mathrm{N}-\mathrm{CH}_{2}$ ), $3.55\left(\mathrm{~s}, 2 \mathrm{H},-\mathrm{CH}_{2}\right), 3.63$ (t, $\left.4 \mathrm{H},-\mathrm{O}-\mathrm{CH}_{2}\right), 6.92-7.84$ $(\mathrm{m}, 4 \mathrm{H}, \mathrm{Ar}-\mathrm{H}) ;{ }^{13} \mathrm{C}$ NMR (DMSO- $\left.d_{6}, \delta \mathrm{ppm}\right): 21.22,48.06,49.92,53.04,54.27,66.26$, 114.34, 126.45, 128.21, 142.36, 149.62, 168.77, 168.20. Anal. calcd. for $\mathrm{C}_{19} \mathrm{H}_{25} \mathrm{~N}_{5} \mathrm{O}_{4}$ : C, 58.90; H, 6.50; N, 18.08; found: C, 59.01; H, 6.58; N, 18.14.

\section{3-[4-(Morpholin-4-yl) phenyl]-4-(1-phenylpiperazin-4-yl methyl)sydnone (6f)}

Yield: 65\%; mp 139-141 ${ }^{\circ} \mathrm{C}$; IR spectrum $\left(\mathrm{KBr}, v, \mathrm{~cm}^{-1}\right)$ : 2918, $2864\left(\mathrm{CH}_{2}\right), 1759(\mathrm{CO}) ;{ }^{1} \mathrm{H}$ NMR (DMSO-d $d_{6}, \delta$ ppm): 2.55 (t, 4H, -N-CH $), 3.22\left(\mathrm{t}, 8 \mathrm{H},-\mathrm{N}-\mathrm{CH}_{2}\right), 3.53\left(\mathrm{~s}, 2 \mathrm{H},-\mathrm{CH}_{2}\right)$, 3.61 (t, $\left.4 \mathrm{H},-\mathrm{O}-\mathrm{CH}_{2}\right), 6.79-7.72$ (m, $\left.9 \mathrm{H}, \mathrm{Ar}-\mathrm{H}\right) ;{ }^{13} \mathrm{C}$ NMR (DMSO- $\left.d_{6}, \delta \mathrm{ppm}\right): 48.09,53.12$, 53.42, 54.62, 66.47, 114.34, 121.90, 126.23, 128.22, 129.68, 142.33, 149.71, 168.10. Anal. calcd. for $\mathrm{C}_{23} \mathrm{H}_{27} \mathrm{~N}_{5} \mathrm{O}_{3}$ : C, 64.54; H, 6.46; N, 16.62; found: C, 64.47; H, 6.52; N, 16.70 .

\section{3-[4-(Morpholin-4-yl) phenyl]-4-(1-ethylpiperazin-4-yl methyl)sydnone (6g)}

Yield: 54\%; mp 118-120 ${ }^{\circ} \mathrm{C}$; IR spectrum $\left(\mathrm{KBr}, v, \mathrm{~cm}^{-1}\right)$ : 2987-2864 $\left(\mathrm{CH}_{2}, \mathrm{CH}_{3}\right), 1742$ (CO); ${ }^{1} \mathrm{H}$ NMR (DMSO-d $d_{6}, \delta$ ppm): 1.09 (t, 3H, $\mathrm{CH}_{3}$ ), 2.39 (q, $2 \mathrm{H}, \mathrm{C}_{2} \mathrm{CH}_{3}$ ), 2.38 (t, 4H, - N-CH $\mathrm{CH}_{2}$ ), 
2.54 (t, 4H, -N-CH $), 3.21$ (t, 4H, -N-CH $), 3.54$ (s, 2H, $-\mathrm{CH}_{2}$ ), 3.60 (t, 4H, -O-CH $\mathrm{CH}_{2}$, 6.717.59 (m, 4H, Ar-H); ${ }^{13} \mathrm{C}$ NMR (DMSO- $d_{6}, \delta$ ppm): 13.76, 48.14, 49.66, 53.03, 54.28, 55.94, 66.36, 109.57, 114.39, 126.31, 128.25, 142.33, 149.79, 168.19. Anal. calcd. for $\mathrm{C}_{19} \mathrm{H}_{27} \mathrm{~N}_{5} \mathrm{O}_{3}$ : C, 60.78; H, 7.79; N, 18.65; found: C, 60.69; H, 7.71; N, 18.73 .

\section{3-[4-(Morpholin-4-yl) phenyl]-4-[1-(4-methoxyphenyl)piperazin-4-yl} methyl)sydnone (6h)

Yield: 62\%; mp 156-158 ${ }^{\circ} \mathrm{C}$; IR spectrum (KBr, v, cm $\left.{ }^{-1}\right)$ : 2988-2855 $\left(\mathrm{CH}_{2}, \mathrm{CH}_{3}\right), 1753$ (CO); ${ }^{1} \mathrm{H}$ NMR (DMSO- $d_{6}, \delta$ ppm): $2.51\left(\mathrm{t}, 4 \mathrm{H},-\mathrm{N}-\mathrm{CH}_{2}\right.$ ), 2.67 (t, $\left.4 \mathrm{H},-\mathrm{N}-\mathrm{CH}_{2}\right), 3.20(\mathrm{t}, 4 \mathrm{H},-\mathrm{N}-$ $\mathrm{CH}_{2}$ ), 3.52 (s, $2 \mathrm{H},-\mathrm{CH}_{2}$ ), 3.61 (t. 4H, O- $\mathrm{CH}_{2}$ ), 3.65 (s, 3H, $\mathrm{CH}_{3}$ ), 6.67-7.53 (m, 4H, Ar-H); ${ }^{13} \mathrm{C}$ NMR (DMSO- $\left.d_{6}, \delta \mathrm{ppm}\right): 45.21,53.00,54.41,57.39,60.39,66.32,114.35,126.45$, 128.32, 142.46, 149.83, 168.26. Anal. calcd. for $\mathrm{C}_{24} \mathrm{H}_{29} \mathrm{~N}_{5} \mathrm{O}_{4}$ : C, 63.84; H, 6.47; N, 15.51; found: C, 63.78; H, 6.52; N, 15.69 .

\section{3-[4-(Morpholin-4-yl) phenyl]-4-(1-methyl-3-phenylpiperazin-4-yl}

methyl)sydnone (6i)

Yield: 58\%; mp 167-169 ${ }^{\circ} \mathrm{C}$; IR spectrum (KBr, v, cm $\left.{ }^{-1}\right)$ : 2965-2854 $\left(\mathrm{CH}_{2}, \mathrm{CH}_{3}\right), 1760$ (CO); ${ }^{1} \mathrm{H}$ NMR (DMSO-d $\left.d_{6}, \delta \mathrm{ppm}\right): 2.23\left(\mathrm{~s}, 3 \mathrm{H}, \mathrm{CH}_{3}\right), 2.71\left(\mathrm{t}, 2 \mathrm{H},-\mathrm{N}_{-} \mathrm{CH}_{2}\right), 2.78\left(\mathrm{t}, 2 \mathrm{H},-\mathrm{N}-\mathrm{CH}_{2}\right)$, 3.17 (t, $4 \mathrm{H},-\mathrm{N}-\mathrm{CH}_{2}$ ), 3.50 (s, 2H, $\mathrm{CH}_{2}$ ), 3.64 (t, 4H, -O-CH $), 4.07$ (t, $\left.1 \mathrm{H},-\mathrm{N}-\mathrm{CH}\right), 6.71-7.66$ (m, 9H, Ar-H); ${ }^{13} \mathrm{C}$ NMR (DMSO- $d_{6}, \delta$ ppm): 45.82, 46.89, 53.02, 54.45, 56.45, 61.26, 66.12, 67.51, 114.28, 126.39, 127.07, 127.99, 128.23, 128.92, 134.85, 142.34, 149.53, 168.15. Anal. calcd. for $\mathrm{C}_{24} \mathrm{H}_{29} \mathrm{~N}_{5} \mathrm{O}_{3}$ : C, 66.19; H, 6.71; N, 16.08; found: C, 66.25; H, 6.76; N, 16.15 .

\section{3-[4-(Morpholin-4-yl) phenyl]-4-[1-(2,3-dichlorophenyl)piperazin-4-yl methyl]sydnone (6j)}

Yield: 63\%; mp 144-146 ${ }^{\circ} \mathrm{C}$; IR spectrum $\left(\mathrm{KBr}, v, \mathrm{~cm}^{-1}\right)$ : 2929, $2863\left(\mathrm{CH}_{2}\right), 1752(\mathrm{CO}) ;{ }^{1} \mathrm{H}$ NMR (DMSO- $d_{6}, \delta$ ppm): 2.61(t, 4H, $\left.-\mathrm{N}_{-} \mathrm{CH}_{2}\right), 3.21\left(\mathrm{t}, 4 \mathrm{H},-\mathrm{N}-\mathrm{CH}_{2}\right), 3.42\left(\mathrm{t}, 4 \mathrm{H},-\mathrm{N}-\mathrm{CH}_{2}\right)$, 3.53 (s, $2 \mathrm{H}, \mathrm{CH}_{2}$ ), 3.63 (t, 4H, -O-CH $)_{2}$, 6.65-7.09 (m, 4H, Ar-H); ${ }^{13} \mathrm{C}$ NMR (DMSO-d 6 , $\delta$ ppm): 48.42, 52.29, 53.01, 54.55, 66.21, 114.24, 117.61, 123.98, 126.46, 127.27, 128.22, 129.29, 133.34, 142,39, 149.51, 150.68, 168.11. Anal. calcd. for $\mathrm{C}_{23} \mathrm{H}_{25} \mathrm{Cl}_{2} \mathrm{~N}_{5} \mathrm{O}_{3}$ : C, 56.33; H, 5.14; N, 14.46; found: C, 56.28; H, 5.09; N, 14.33 .

\section{3-[4-(Morpholin-4-yl) phenyl] sydnone sulphonyl chloride (7)}

Chlorosulphonic acid $(0.03 \mathrm{~mol})$ was added drop wise in to the mixture of 3-[4-(morpholin-4-yl) phenyl] sydnones $(0.01 \mathrm{~mol})$ and catalytic amount of $\mathrm{P}_{2} \mathrm{O}_{5}$ over 30 min with constant stirring at $0-5{ }^{\circ} \mathrm{C}$. When all the chlorosulphonic acid has been added (about $1 \mathrm{~h}$ ), stir the reaction mixture for $2 \mathrm{~h}$ and keep it overnight at room temperature or heat the reaction mixture on a water bath for 1 hour to complete the reaction. Allow to cool and pour the oily mixture into crushed ice with stirring. Break up any lumps of solid material and stir the mixture for several minutes in order to obtain greenish-yellow solid product. Filter of the product and wash with cold water and dry to give compound 7. Yield 87.00\%; mp 205-207 ${ }^{\circ} \mathrm{C}$. IR spectrum $\left(\mathrm{KBr}, \mathrm{v}, \mathrm{cm}^{-1}\right)$ : 1755 (CO), 1396, $1178\left(\mathrm{SO}_{2}\right) ;{ }^{1} \mathrm{H}$ NMR $\left(\mathrm{CDCl}_{3}, \delta \mathrm{ppm}\right): 3.18$. (t, $\left.4 \mathrm{H},-\mathrm{N}-\mathrm{CH}_{2}\right), 3.63(\mathrm{t}, 4 \mathrm{H},-\mathrm{O}-$ $\mathrm{CH}_{2}$ ), 6.77-7.69 (m, 4H, Ar-H). Anal. calcd. for $\mathrm{C}_{12} \mathrm{H}_{14} \mathrm{~N}_{3} \mathrm{O}_{5} \mathrm{~S}_{2} \mathrm{Cl}$ : C, 41.42; H, 4.06; N, 12.07; S, 9.23; found: C, 41.41; H, 4.18; N, 12.15; S, 9.14.

\section{Synthesis of compounds $(\mathbf{8} \boldsymbol{a}-\mathbf{j})$}

3-[4-(Morpholin-4-yl) phenyl] sydnone sulphonyl chloride $(0.011 \mathrm{~mol})$ was dissolved in acetone. A solution of various secondary amine $(0.022 \mathrm{~mol})$ in acetone was added drop wise in to 3-[4-(morpholin-4-yl) phenyl] sydnone sulphonyl chloride solution over a period of $5 \mathrm{~h}$ 
with constant stirring. Add $1.0 \mathrm{~mL}$ of pyridine to the well stirred solution after $1 \mathrm{~h}$ and $2 \mathrm{~h}$ respectively during the reaction. The solution was poured in to ice with stirring. Precipitation was collected by filtration, washed thrice with water and dried. recrystallization from benzene to give compounds $\mathbf{8}(\mathbf{a}-\mathbf{j})$.

\section{3-[4-(Morpholin-4-yl) phenyl]- 4-(piperazin-4-yl sulfonyl)sydnone (8a)}

Yield: 2.39 g (64\%); mp 192-194 ${ }^{\circ} \mathrm{C}$; IR spectrum $\left(\mathrm{KBr}, v, \mathrm{~cm}^{-1}\right)$ : 3225 (NH), 1760 (CO), 1351, $1185\left(\mathrm{SO}_{2}\right) ;{ }^{1} \mathrm{H}$ NMR (DMSO- $\left.d_{6}, \delta \mathrm{ppm}\right): 1.91(\mathrm{~s}, 1 \mathrm{H}, \mathrm{NH}), 2.46\left(\mathrm{t}, 4 \mathrm{H},-\mathrm{N}-\mathrm{CH}_{2}\right), 2.65$ (t, 4H, -N-CH $), 3.19\left(\mathrm{t}, 4 \mathrm{H},-\mathrm{N}-\mathrm{CH}_{2}\right), 3.64\left(\mathrm{t}, 4 \mathrm{H},-\mathrm{O}-\mathrm{CH}_{2}\right), 6.79-7.72(\mathrm{~m}, 4 \mathrm{H}, \mathrm{Ar}-\mathrm{H}) ;{ }^{13} \mathrm{C}$ NMR (DMSO- $d_{6}, \delta$ ppm): 44.51, 51.03, 53.43, 66.29, 109.36, 114.35, 126.35, 128.03, 149.61, 169.19. Anal. calcd. for $\mathrm{C}_{16} \mathrm{H}_{21} \mathrm{~N}_{5} \mathrm{O}_{5} \mathrm{~S}$ : C, 48.29; H, 5.61; N, 17.63; found: C, 48.35; H, 5.70; N, 17.55.

\section{3-[4-(Morpholin-4-yl) phenyl]-4-(morpholin-4-yl sulfonyl)sydnone (8b)}

Yield: 2.39 g (70\%); mp 247-249 ${ }^{\circ} \mathrm{C}$; IR spectrum (KBr, $\left.v, \mathrm{~cm}^{-1}\right)$ : 1755 (CO), 1370, 1182 $\left(\mathrm{SO}_{2}\right) ;{ }^{1} \mathrm{H}$ NMR (DMSO- $\left.d_{6}, \delta \mathrm{ppm}\right): 2.93\left(\mathrm{t}, 4 \mathrm{H},-\mathrm{N}-\mathrm{CH}_{2}\right), 3.22\left(\mathrm{t}, 4 \mathrm{H},-\mathrm{N}-\mathrm{CH}_{2}\right), 3.65(\mathrm{t}, 8 \mathrm{H}$, $-\mathrm{O}_{-} \mathrm{CH}_{2}$ ), 6.86-7.70 (m, 4H, Ar-H); ${ }^{13} \mathrm{C}$ NMR (DMSO- $\left.d_{6}, \delta \mathrm{ppm}\right): 47.51,53.28,66.29$, 109.36, 114.31, 126.35, 128.08, 149.61, 169.19. Anal. calcd. for $\mathrm{C}_{16} \mathrm{H}_{20} \mathrm{~N}_{4} \mathrm{O}_{6} \mathrm{~S}: \mathrm{C}, 48.35 ; \mathrm{H}$, 5.37; N, 13.88; found: C, 48.25; H, 5.45; N, 13.95 .

\section{3-[4-(Morpholin-4-yl) phenyl]-4-(1-methylpiperazin-4-yl sulfonyl)sydnone (8c)}

Yield: 2.39 g (72\%); mp 218-220 ${ }^{\circ} \mathrm{C}$; IR spectrum (KBr, v, cm $\left.{ }^{-1}\right)$ : 2989, $2875\left(\mathrm{CH}_{3}\right), 1758$ (CO), 1386, $1177\left(\mathrm{SO}_{2}\right) ;{ }^{1} \mathrm{H}$ NMR (DMSO-d $\left.6, \delta \mathrm{ppm}\right): 2.25$ (s, 3H, $\left.\mathrm{CH}_{3}\right), 2.36(\mathrm{t}, 4 \mathrm{H},-\mathrm{N}-$ $\left.\mathrm{CH}_{2}\right), 2.45\left(\mathrm{t}, 4 \mathrm{H},-\mathrm{N}-\mathrm{CH}_{2}\right), 3.19\left(\mathrm{t}, 4 \mathrm{H},-\mathrm{N}-\mathrm{CH}_{2}\right), 3.66\left(\mathrm{t}, 4 \mathrm{H},-\mathrm{O}-\mathrm{CH}_{2}\right), 6.79-7.73(\mathrm{~m}, 4 \mathrm{H}$, Ar-H); ${ }^{13} \mathrm{C}$ NMR (DMSO- $d_{6}, \delta$ ppm): 46.81, 48.47, 53.27, 56.23, 66.34, 109.43, 114.23, 126.38, 128.12, 149.78, 169.12. Anal. calcd. for $\mathrm{C}_{17} \mathrm{H}_{23} \mathrm{~N}_{5} \mathrm{O}_{5} \mathrm{~S}$ : C, 49.42; H, 6.12; N, 16.87; found: C, 49.55; H, 6.05; N, 16.95 .

\section{3-[4-(Morpholin-4-yl) phenyl]-4-(piperidin-4-yl sulfonyl)sydnone (8d)}

Yield: 2.39 g (80\%); mp 206-208 ${ }^{\circ} \mathrm{C}$; IR spectrum (KBr, v, $\left.\mathrm{cm}^{-1}\right)$ : 1752 (CO), 1370, 1179 $\left(\mathrm{SO}_{2}\right) ;{ }^{1} \mathrm{H}$ NMR (DMSO-d $\left.{ }_{6}, \delta \mathrm{ppm}\right): 1.53-1.59\left(\mathrm{~m}, 6 \mathrm{H}, 3 \mathrm{CH}_{2}\right), 3.04\left(\mathrm{t}, 4 \mathrm{H},-\mathrm{N}-\mathrm{CH}_{2}\right), 3.20(\mathrm{t}, 4 \mathrm{H}$, $-\mathrm{N}-\mathrm{CH}_{2}$ ), 3.63 (t, 4H, -O-CH $\mathrm{CH}_{2}$, 6.76-7.67 (m, 4H, Ar-H); ${ }^{13} \mathrm{C}$ NMR (DMSO-d $d_{6}, \delta \mathrm{ppm}$ ): 24.01, 24.87, 49.23, 53.35, 66.29, 109.29, 114.25, 126.46, 128.23, 149.87, 169.24. Anal. calcd. for $\mathrm{C}_{17} \mathrm{H}_{22} \mathrm{~N}_{4} \mathrm{O}_{5} \mathrm{~S}$ : C, 51.32; H, 5.61; N, 14.20; found: C, 51.42; H, 5.95; N, 14.12.

\section{3-[4-(Morpholin-4-yl) phenyl]-4-(1-acetylpiperazin-4-yl sulfonyl)sydnone(8e)}

Yield: 2.39 g (68\%); mp 212-214 ${ }^{\circ} \mathrm{C}$; IR spectrum (KBr, v, $\left.\mathrm{cm}^{-1}\right)$ : 2987, $2860\left(\mathrm{CH}_{3}\right), 1750$ (CO), 1680 (CO), 1382, $1174\left(\mathrm{SO}_{2}\right) ;{ }^{1} \mathrm{H}$ NMR (DMSO-d $\left.d_{6}, \delta \mathrm{ppm}\right): 2.13$ (s, 3H, $\left.\mathrm{CH}_{3}\right), 2.61$ (t, 4H, -N-CH $), 3.19$ (t, 4H, - $-\mathrm{CH}_{2}$ ), 3.45 (t, 4H, $\left.-\mathrm{N}-\mathrm{CH}_{2}\right), 3.64$ (t, 4H, $\left.-\mathrm{O}-\mathrm{CH}_{2}\right), 6.89-7.74$ (m, 4H, Ar-H); ${ }^{13} \mathrm{C}$ NMR (DMSO-d 6 , $\delta$ ppm): 21.18, 48.03, 49.89, 53.37, 66.21, 109.45, 114.27, 126.41, 128.19, 149.65, 168.78, 169.22. Anal. calcd. for $\mathrm{C}_{18} \mathrm{H}_{23} \mathrm{~N}_{5} \mathrm{O}_{6} \mathrm{~S}: \mathrm{C}, 49.07 ; \mathrm{H}$, 5.59; N, 16.06; found: C, 49.15; H, 5.65; N, 15.95.

\section{3-[4-(Morpholin-4-yl) phenyl]-4-(1-phenylpiperazin-4-yl sulfonyl)sydnone (8f)}

Yield: 2.39 g (82\%); mp 218-220 ${ }^{\circ} \mathrm{C}$; IR spectrum (KBr, v, $\left.\mathrm{cm}^{-1}\right)$ : 1758 (CO), 1375, 1171 $\left(\mathrm{SO}_{2}\right) ;{ }^{1} \mathrm{H}$ NMR (DMSO-d $\left.{ }_{6}, \delta \mathrm{ppm}\right): 2.57$ (t, $4 \mathrm{H},-\mathrm{N}-\mathrm{CH}_{2}$ ), 3.18 (t, 8H, -N-CH $), 3.65(\mathrm{t}, 4 \mathrm{H}$, $-\mathrm{O}-\mathrm{CH}_{2}$ ), 6.79-7.72 (m, 9H, Ar-H); ${ }^{13} \mathrm{C}$ NMR (DMSO-d $d_{6}, \delta \mathrm{ppm}$ ): 48.12, 53.15, 53.39, 66.45, 109.42, 114.28, 121.87, 126.43, 128.19, 129.63, 149.78, 169.12. Anal. calcd. for $\mathrm{C}_{22} \mathrm{H}_{25} \mathrm{~N}_{5} \mathrm{O}_{5} \mathrm{~S}$ : C, 55.79; H, 5.61; N, 14.72; found: C, 55.85; H, 5.65; N, 14.65 . 


\section{3-[4-(Morpholin-4-yl) phenyl]-4-(1-ethylpiperazin-4-yl sulfonyl)sydnone (8g)}

Yield: 2.39 g (75\%); mp 195-197 ${ }^{\circ} \mathrm{C}$; IR spectrum (KBr, v, cm $\left.{ }^{-1}\right)$ : 2964, $2870\left(\mathrm{CH}_{3}\right), 1747$ (CO), 1388, $1177\left(\mathrm{SO}_{2}\right) ;{ }^{1} \mathrm{H}$ NMR (DMSO-d $\left.6, \delta \mathrm{ppm}\right): 1.23\left(\mathrm{t}, 3 \mathrm{H}, \mathrm{CH}_{3}\right), 2.39(\mathrm{q}, 2 \mathrm{H}$, $\mathrm{C}_{2} \mathrm{CH}_{3}$ ), 2.36 (t, 4H, -N-CH ), 2.51 (t, 4H, -N-CH ${ }_{2}$ ), 3.19 (t, 4H, $\left.-\mathrm{N}-\mathrm{CH}_{2}\right), 3.63$ (t, 4H, -O$\mathrm{CH}_{2}$ ), 6.74-7.69 (m, $\left.4 \mathrm{H}, \mathrm{Ar}-\mathrm{H}\right) ;{ }^{13} \mathrm{C}$ NMR (DMSO-d $\left.{ }_{6}, \delta \mathrm{ppm}\right): 13.79,48.10,49.63,53.22$, 53.93, 66.33, 109.54, 114.38, 126.35, 128.25, 149.69, 169.09. Anal. calcd. for $\mathrm{C}_{18} \mathrm{H}_{25} \mathrm{~N}_{5} \mathrm{O}_{5} \mathrm{~S}$ : C, 50.78; H, 6.32; N, 16.29; found: C, 50.85; H, 6.25; N, 16.38 .

\section{3-[4-(Morpholin-4-yl) phenyl]-4-[1-(4-methoxyphenyl)piperazin-4-yl sulfonyl)sydnone (8h)}

Yield: 2.39 g (85\%); mp 187-189 ${ }^{\circ} \mathrm{C}$; IR spectrum (KBr, v, cm $\left.{ }^{-1}\right)$ : 2980, $2858\left(\mathrm{CH}_{3}\right), 1749$ (CO), 1364, $1174\left(\mathrm{SO}_{2}\right)$; ${ }^{1} \mathrm{H}$ NMR (DMSO-d 6 , $\left.\delta \mathrm{ppm}\right): 2.59$ (t, 4H, -N-CH ), $2.66(\mathrm{t}, 4 \mathrm{H},-\mathrm{N}-$ $\mathrm{CH}_{2}$ ), 3.20 (t, 4H, -N-CH $)$, 3.63 (t, 4H, O-CH $\mathrm{CH}_{2}$ ), 3.68 (s, 3H, $\left.\mathrm{CH}_{3}\right), 6.77-7.63(\mathrm{~m}, 4 \mathrm{H}, \mathrm{Ar}-\mathrm{H})$;

${ }^{13} \mathrm{C}$ NMR (DMSO- $\left.d_{6}, \delta \mathrm{ppm}\right): 45.14,53.41,57.33,60.40,66.25,109.36,114.21,126.42$, 128.32, 149.85, 169.25. Anal. calcd. for $\mathrm{C}_{23} \mathrm{H}_{27} \mathrm{~N}_{5} \mathrm{O}_{6} \mathrm{~S}$ : C, 54.82; H, 5.73; N, 13.80; found: C, 54.90; H, 5.68; N, 13.88.

\section{3-[4-(Morpholin-4-yl) phenyl]-4-(1-methyl-3-phenylpiperazin-4-yl sulfonyl)sydnone (8i)}

Yield: 2.39 g (82\%); mp 237-239 ${ }^{\circ} \mathrm{C}$; IR spectrum (KBr, v, cm $\left.{ }^{-1}\right)$ : 2968, $2852\left(\mathrm{CH}_{3}\right), 1755$ (CO), 1380, $1181\left(\mathrm{SO}_{2}\right) ;{ }^{1} \mathrm{H}$ NMR (DMSO-d $\left.6, \delta \mathrm{ppm}\right): 2.25$ (s, 3H, $\left.\mathrm{CH}_{3}\right), 2.71(\mathrm{t}, 2 \mathrm{H},-\mathrm{N}-$ $\left.\mathrm{CH}_{2}\right), 2.78\left(\mathrm{t}, 2 \mathrm{H},-\mathrm{N}-\mathrm{CH}_{2}\right), 3.18\left(\mathrm{t}, 4 \mathrm{H},-\mathrm{N}-\mathrm{CH}_{2}\right), 3.65\left(\mathrm{t}, 4 \mathrm{H},-\mathrm{O}-\mathrm{CH}_{2}\right), 4.10(\mathrm{t}, 1 \mathrm{H},-\mathrm{N}-\mathrm{CH})$, 6.75-7.67 (m, 9H, Ar-H); ${ }^{13} \mathrm{C}$ NMR (DMSO- $d_{6}, \delta$ ppm): 45.83, 46.91, 53.45, 56.53, 61.24, 66.07, 66.52, 109.26, 114.22, 126.35, 127.00, 127.98, 128.22, 128.95, 134.84, 149.58, 169.17. Anal. calcd. for $\mathrm{C}_{23} \mathrm{H}_{27} \mathrm{~N}_{5} \mathrm{O}_{5} \mathrm{~S}$ : C, 56.45; H, 6.06; N, 14.32; found: C, 56.55; H, 5.97; N, 14.28 .

\section{3-[4-(Morpholin-4-yl) phenyl]-4-[1-(2,3-dichlorophenyl)piperazin-4-yl} sulfonyl] sydnone (8j)

Yield: 2.39 g (74\%); mp 217-219 ${ }^{\circ} \mathrm{C}$; IR spectrum (KBr, v, $\left.\mathrm{cm}^{-1}\right): 1760$ (CO), 1374, 1172 $\left(\mathrm{SO}_{2}\right) ;{ }^{1} \mathrm{H}$ NMR (DMSO- $\left.d_{6}, \delta \mathrm{ppm}\right): 2.56\left(\mathrm{t}, 4 \mathrm{H},-\mathrm{N}-\mathrm{CH}_{2}\right), 3.20\left(\mathrm{t}, 8 \mathrm{H},-\mathrm{N}-\mathrm{CH}_{2}\right), 3.63(\mathrm{t}, 4 \mathrm{H}$, $-\mathrm{O}_{-} \mathrm{CH}_{2}$ ), 6.58-7.11 (m, 7H, Ar-H); ${ }^{13} \mathrm{C}$ NMR (DMSO- $\left.d_{6}, \delta \mathrm{ppm}\right): 48.44,52.26,53.55$, 66.23, 109.16, 114.29, 117.62, 123.96, 126.43, 127.25, 128.20, 129.23, 133.33, 149.50, 150.63, 169.10. Anal. calcd. for $\mathrm{C}_{22} \mathrm{H}_{23} \mathrm{Cl}_{2} \mathrm{~N}_{5} \mathrm{O}_{5} \mathrm{~S}$ : C, 48.59; H, 4.61; N, 12.88; found: C, 48.65; H, 4.58; N, 12.96.

\section{Results and Discussion}

\section{Biological evaluation (Antimicrobial activity)}

All the newly synthesized compounds were screened in vitro for their antibacterial and antifungal activities by broth dilution method (Table 1). The antibacterial activity of the compounds was tested against S.aureus and B.subtilis as Gram positive and P.aeruginosa and E.coli as Gram negative bacterial strains. Antifungal activity of the compound was tested against C.albicans as fungal strain. Ciprofloxacin was used as standard antibacterial drug and Flucanazole was used as standard antifungal drug. Standard strains were procured from Institute of Microbial Technology, Chandigarh.

The lowest concentration inhibiting growth of the organism is recorded as the MIC. DMSO was used as diluent. The stock $1000 \mu \mathrm{g} / \mathrm{mL}$ was prepared. Serial dilutions were prepared in primary and secondary screening. Mueller Hinton Broth was used as nutrient 
medium to grow and dilute the drug suspension for the test bacteria, and sabaouraus dextrose broth used for fungal nutrition. Inoculum size for test strain was adjusted to $10^{8} \mathrm{CFU}$ [Colony Forming Unit] per milliliter by comparing the turbidity. The control tube containing no antibiotic is immediately sub cultured (before inoculation) by spreading a loopful evenly over a quarter of plate of medium suitable for the growth of the test organism and put for incubation at $37^{\circ} \mathrm{C}$ overnight. The tubes are then incubated overnight. The MIC of the control organism is read to check the accuracy of the drug concentrations. The amount of growth from the control tube before incubation (which represents the original inoculum) is compared.

Table 1. Antimicrobial activity (MIC $\mu \mathrm{g} / \mathrm{mL}$ ) of synthesized compounds 6(a-j) and 8(a-j)

\begin{tabular}{|c|c|c|c|c|c|}
\hline \multirow{3}{*}{ Compd. } & \multicolumn{5}{|c|}{ Minimum inhibitory concentration, $\mu \mathrm{g} / \mathrm{mL}$} \\
\hline & \multicolumn{2}{|c|}{ Gram +ve } & \multicolumn{2}{|c|}{ Gram -ve } & \multirow{2}{*}{$\begin{array}{l}\text { Antifungal } \\
\text { C.albicans } \\
\end{array}$} \\
\hline & S.aureus & B. subtilis & P.aeruginosa & E.coli & \\
\hline 6a & 1000 & 500 & 500 & 500 & 500 \\
\hline $6 \mathbf{b}$ & 500 & 250 & 1000 & 1000 & 500 \\
\hline 6c & 250 & 200 & 200 & 500 & 1000 \\
\hline 6d & 100 & 100 & 500 & 250 & 200 \\
\hline $6 e$ & 500 & 500 & 1000 & 500 & 250 \\
\hline $6 f$ & 500 & 500 & 200 & 500 & 500 \\
\hline $6 \mathbf{g}$ & 500 & 500 & 1000 & 500 & 1000 \\
\hline $6 \mathbf{6}$ & 200 & 250 & 250 & 500 & 1000 \\
\hline $6 \mathbf{i}$ & 62.5 & 500 & 500 & 500 & 500 \\
\hline 6j & 250 & 500 & 1000 & 1000 & 500 \\
\hline 8a & 100 & 100 & 200 & 500 & 200 \\
\hline $8 \mathbf{b}$ & 250 & 500 & 500 & 500 & 500 \\
\hline $8 c$ & 62.5 & 62.5 & 100 & 100 & 250 \\
\hline 8d & 200 & 100 & 200 & 100 & 500 \\
\hline $\mathbf{8 e}$ & 250 & 500 & 500 & 500 & 1000 \\
\hline $8 f$ & 500 & 500 & 1000 & 1000 & 1000 \\
\hline $8 g$ & 500 & 500 & 1000 & 1000 & 1000 \\
\hline 8h & 250 & 250 & 500 & 250 & 250 \\
\hline $8 \mathbf{i}$ & 500 & 500 & 500 & 500 & 500 \\
\hline $8 \mathbf{j}$ & 500 & 500 & 250 & 500 & 500 \\
\hline Ciprofloxacin & 50 & 50 & 25 & 25 & --- \\
\hline Flucanazole & --- & --- & --- & --- & 100 \\
\hline
\end{tabular}

From the screening results, it can be seen that compound $\mathbf{6 d}$ showed good activity against gram +ve bacteria. Compound 8c showed excellent activity against all bacteria, whereas Compound 8a displayed good activity against both of the Gram positive bacteria. Compound 6i was found significantly active against Gram positive bacteria S.aureus compared with Ciprofloxacin. Compound 8d showed good activity against Gram positive bacteria S.aureus and Gram negative bacteria E.coli. The tested compound 6d and 8a demostrated good antifungal activity against C.albicans. Rest of the compounds showed moderate to weak activity against other bacteria and fungi compared with the standard drugs.

\section{Conclusion}

In this work, we have synthesized a series of new sydnone derivatives $\mathbf{6 ( a - j )}$ and $\mathbf{8 ( a - j )}$ which were tested for their antimicrobial activity and their structures are confirmed successfully by IR, ${ }^{1} \mathrm{H}$ NMR, ${ }^{13} \mathrm{C}$ NMR spectra and elemental analysis. Antibacterial activity 
of title compounds showed that methyl group present at $4^{\text {th }}$ position of piperazine ring in compound 8c could be responsible for increase activity against S.aureus, B.subtilis, P.aeruginosa and E.coli. Compound $\mathbf{6 i}$ has phenyl group at $2^{\text {nd }}$ position and methyl group at $4^{\text {th }}$ position to show highest activity against S.aureus. The activity varies with the different substituents on methylene and sulphonyl linkages.

Acknowledgment

The authors would like to acknowledge Professor and Head Dr. P. Bahadur, Department of Chemistry, Veer Narmad South Gujarat University, Surat for providing laboratory facilities and D. Rajani, Microcare Laboratory, Surat, for help in antimicrobial activity work. The authors also thank to SAIF, Chandigarh for analytical analysis.

\section{References}

1. Asundaria S T and Patel K C, Synth Commun., 2010, 40, 1899-1906.

2. Mishra L, Said M K, Itokawa H and Takeya K, Bioorg Med Chem., 1995, 3(9), 1241.

3. Suman S P and Bahel S C, J Indian Chem Soc., 1979, 56, 712.

4. Badami B V and Puranik G S, Rev Roum Chim., 1982, 27, 281.

5. Greco C V and Mehta J R, J Heterocycl Chem., 1979, 16, 1059-1060.

6. Ito S and Turnbull K, Synth Commun., 1996, 26, 1441.

7. Tien H J, Yeh M Y and Hwang C Y, J Chin Chem Soc., 1985, 32, 461.

8. Tien H J, Lin S T and Sheu I T, Can J Chem., 1994, 72, 1610.

9. Turnbull K and George J C, Synth Commun., 1996, 26, 2757.

10. Tien H J and Ohta M, Bull Chem Soc Jpn., 1972, 45, 2944.

11. Vasileva V F and Yashunskii V G, Khim Nauk Prom., 1958, 3, 282; Chem Abstr., 1958, 52, 20013.

12. Tien H J and Lee Y K, J Chin Chem Soc., 1988, 35(1), 63-69.

13. Guhring H, Hamza M, Sergejeva M, Ates M, Kotalla C E, Ledent C and Brune K, Euro J Pharm., 2002, 454, 153-163.

14. Kier L B and Dhawan D, J Pharma Sci., 1962, 51, 1058.

15. Kamble R R, Latthe P R and Badami B V, Heteroatom Chem., 2007, 18, 50-54.

16. Kier L B and Roche E B, J Pharm Sci., 1967, 56, 149-168.

17. Moustafa M A, Gineinah M M, Nasr M N and Bayoumi W A, Arch Pharm., 2004, 337, 427.

18. Dunkley C S and Thoman C J, Bioorg Med Chem Lett., 2003, 13(17), 2899-2901.

19. Hutchinson I, Jennings S A, Vishnuvajjala B R, Westwell A D and Stevens M F G, $J$ Med Chem., 2002, 45, 744-747.

20. Kavali J R and Badami B V, IL Farmaco., 2000, 55(5), 406-409.

21. Bekhit A A, Habib N S and E-Din A, Boll Chim Farm., 2001, 140, 297.

22. Nyberg W H and Cheng C C, J Med Chem., 1965, 8(4), 531-533.

23. Popoff I C and Singhal G H, J Med Chem., 1968, 11, 631.

24. Mc-Caustland D J, Burton W H and Cheng C C, J Heterocycl Chem., 1971, 8, 89-97.

25. Pala G, Mantegani A, Coppi G and Geniova R, Chim Ther., 1969, 4, 312; Chem Abstr., 1969, 71, 3328q.

26. Satyanarayana K and Rao M N A, Eur Med Chem., 1995, 30, 641.

27. Hsiu S M and Fang-Ying K, Bioorg Med Chem., 2004, 12, 4633-4643.

28. Wagner H and Hill J B, J Med Chem., 1974, 17, 1337.

29. Tien H J, Tsai Y H, Yeh W Y, Yeh J C, Lee Y K and Young S H, J Chin Chem Soc., 1990, 37, 79-84.

30. Chan W L, Yana H and Szeto Y S, Mat Lett., 2004, 58, 882.

31. Geoffroy I, Carre B, Lemordant D, Herreyre S and Biensan P, ITS Lett Batt New Tech Med., 2000, 1, 20. 